\title{
Multihop Transmission Protocol Using Cooperative Diversity over Rayleigh Fading Channel
}

\author{
Tran Trung Duy $\cdot$ Jong-Soo Kim
}

\begin{abstract}
In this paper, we propose a novel cooperative routing protocol (NCRP) for wireless networks. The proposed protocol uses cooperative transmission to improve end-to-end outage probability. The broadcast nature ensures that the destination can receive a packet from the source or from the relays and if it cannot correctly decode the packet, the successful relays will start a retransmission. The NCRP protocol can skip some transmissions from the intermediate relays, thereby reducing the total power consumption. Theoretical results are derived and verified by simulation results.

Key words: Diversity, Multihop Network, Rayleigh Fading Channel, Outage Probability, Relay Channel.
\end{abstract}

\section{I . Introduction}

A number of cooperative transmission protocols have been presented in the current literature [1] [3], but most of the recent work related to cooperative communication has been performed on single-hop networks. These protocols use the source to broadcast the signal and the relays then receive, decode, and forward the signal to the destination. However, the multi-hop relaying scheme, where a source communicates with a destination via a number of relays, is now viewed as a promising technique for application in current and future wireless systems. In the conventional multi-hop transmission, each terminal on the line route processes only the signal received from its previous terminal and forwards that signal to next terminal [4]. The concept of the multi-hop diversity protocols [5] [8] has recently been introduced. In these protocols, the broadcast nature of the wireless channel allows each relay on the primary route to receive signals from all previous terminals. The relay combines the signal appropriately, decodes it, and then re-transmits the decoded signal. Nevertheless, this type of strategy requires each node to restore all versions of the received signals, which requires a huge storage capacity. The power consumption and delay time, which are important criteria in multi-hop transmission, are not also considered.

In this paper, we propose a novel cooperative routing protocol (NCRP) in order to achieve better performance in terms of end-to-end outage probability. Our goal was also to reduce the average number of transmissions needed for direct transmission protocols. We assume that a route exists between the source and the destination, which has been established by the network layer.

Similar to [5] [8], due to the broadcast nature of a wireless medium, each node on the route can receive packets transmitted by its previous nodes. If the destination does not successfully decode the packet, a retransmission is required. Some of the relays that can successfully receive the packet will then be chosen to re-transmit the packet to the destination. We derive the exact expressions of the end-to-end outage probability and the average number of transmissions and verify them by Monte Carlo simulations. Experimental results confirm that the proposed protocol outperforms the direct transmission protocols in the high signal-to-noise ratio (SNR) region.

The rest of the paper is organized as follows. The system model and outage analysis are described in Section II and Section III, respectively. Section IV presents the simulation results and Section V concludes the paper.

\section{System Model}

Fig. 1 shows an $M$-hop wireless network operating

Manuscript received November 2, 2011 ; Revised February 7, 2012 ; Accepted February 24, 2012. (ID No. 20111102-027J)

Department of Electronic Engineering, University of Ulsan, Ulsan, Korea.

Corresponding Author : Jong-Soo Kim (e-mail : jongsoo@mail.ulsan.ac.kr)

This is an Open-Access article distributed under the terms of the Creative Commons Attribution Non-Commercial License (http:/creativecommons.org/licenses/ by-nc/3.0) which permits unrestricted non-commercial use, distribution, and reproduction in any medium, provided the original work is properly cited. 


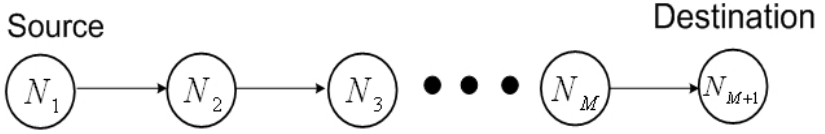

Fig. 1. An $M$-hop route is established by the network layer.

over independent Rayleigh fading channels. The source $N_{1}$ communicates with the destination node $N_{M+1}$ via $M-1$ relay nodes denoted as $N_{2}, N_{3}, \cdots, N_{M}$. The relay nodes are numbered with respect to their distance to the destination; i.e., the relay $N_{M}$ is nearest and the relay $N_{2}$ is the furthest. The assumptions used in this paper are as follows:

i. The channels between any two nodes are subjected to block and flat Rayleigh fading plus AWGN.

ii. Each node has a single half duplex radio and a single antenna. Hence, for medium access, a timedivision channel allocation is occupied in order to realize orthogonal channels.

iii. Receivers can use maximal ratio combining (MRC) to combine the received packets.

iv. Each node on the route has a routing table, which includes the routing information; e.g., the ID of nodes on the route, its position on the route, etc.

v. The packet is attached with cyclic redundancy check (CRC) and the CRC is assumed to be perfect.

vi. The packet has an overhead part and a load part. Each transmitter will include the ID of the intended receiver in the overhead part. The overhead part is also assumed to use powerful error-correcting codes (ECC) so that it can be received without errors at far distances.

vii. Control signals such as acknowledgment (ACK) and negative acknowledgment (NACK) also use powerful ECC for error-free reception at all nodes on the established route.

viii. The size of control signals is small compared to the size of the packet. Therefore, the delay time and transmit power for transmitting them can be ignored compared with those needed for transmitting the packet.

The operation of our proposal is as follows:

At the first stage, the source broadcasts its packet, which can be received by the destination and all of the relays, which then attempt to decode the received packet.

After finishing the first stage, if the destination decodes correctly, it sends an ACK signal to inform de- coding status. In this case, the source will start to transmit a new packet. Otherwise, the destination sends a NACK message to request a retransmission.

After receiving the destination's NACK message, the relays transmit the control signal to inform the decoding status. Successful relays transmit an ACK signal while unsuccessful ones send a NACK signal. The control signals are assumed to be separated by time-division technique and they are also received by all the nodes without errors. The successful relays then will re-transmit the packet to the destination at the second stage in a predetermined order; e.g., a node with low ID or nearer to the destination will transmit first. The destination and the unsuccessful relays receive the transmitted packets and decode them using the MRC technique.

Now, if the destination again does not correctly decode, the operation goes to the third stage. Similar to the second stage, the relays transmit the control signal to inform the decoding status. Note that each relay is only allowed to transmit the packet to the destination once. Therefore, the relays that were successful in the second stage will not send a control message, nor will they receive or decode the packet again. Next, the relays that are successful at this stage will re-transmit the packet to the destination.

This procedure only terminates in two cases, as follows: i) the destination receives the packet correctly ii) at the end of any stage, the destination and relays cannot decode the packet successfully.

In order to avoid a huge storage, all of the nodes, including the destination and relays, will release the received packets from their buffers after each stage.

In the conventional direct transmission (DT) protocol, the packet is relayed hop-by-hop from the source to the destination. Therefore, a successful packet transmission in this protocol is realized through $M$ stages.

For a clear illustration of our proposal, we consider an example as shown in Figs. (2) (4). We consider a 6-hop route with three stages of the data transmission. At the first stage, the source $N_{1}$ broadcasts the packet to the destination $N_{7}$ and all of the relays. Because the destination is unable to decode the packet successfully, relays $N_{2}$ and $N_{4}$, which can successfully decode the packet, retransmit it to the destination at the second stage. Again, due to the unsuccessful decoding at the destination, the operation goes to next stage. At the third stage, we assume that only relay $N_{5}$ can re-transmit the packet to the destination. Next, because the destination can successfully decode the packet received from relay $N_{5}$, the data transmission ends here.

We can observe that the proposed protocol uses three 


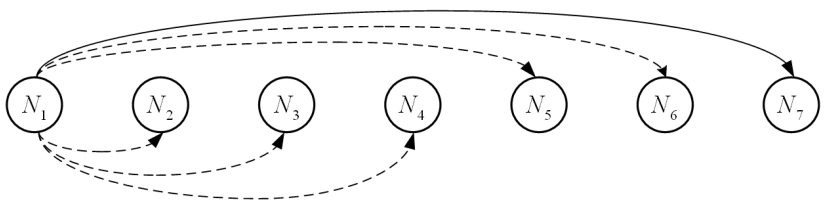

Fig. 2. The source broadcasts the packet.

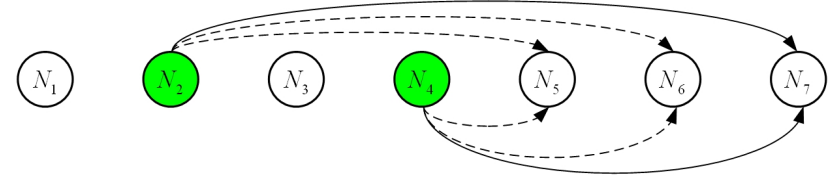

Fig. 3. The relays $N_{2}$ and $N_{4}$ transmit the packet to the destination at the second stage.
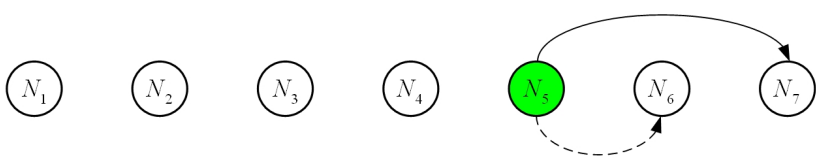

Fig. 4. The relay $N_{5}$ transmits the packet to the destination at the third stage.

stages, with 4 nodes (i.e., $N_{1}, N_{2}, N_{4}$, and $N_{5}$ ) taking part in the data transmission, while the DT protocol uses 6 stages and 6 nodes for the transmission of the packet. Hence, the NCRP protocol can reduce the number of transmissions and reduce power consumption.

\section{Outage Analysis}

\section{3-1 Rayleigh Fading Channel}

The signal received at node $j$ due to the transmission of node $i$ is given by

$$
r_{i, j}(n)=\sqrt{P} h_{i, j} s+\eta_{j}(n)
$$

where $P$ is the average transmitted power, $\eta_{j}(n)$ is AWGN noise sample with variance $N_{0} / 2$ per dimension at terminal $j, h_{i, j}$ is fading coefficient between node $i$ and node $j, s$ is the packet transmitted by node $i$.

We can take path loss into account by modeling the variance of channel coefficient between node $i$ and node $j$ as a function of distance between two nodes [9] as

$$
\sigma_{i, j}^{2}=d_{i, j}{ }^{-\beta}
$$

where $\beta$ is path loss exponent that varies from 2 to 6 and $d_{i, j}$ is the distance between node $i$ and node $j$.

In this paper, we assume that the packet is decoded successfully if the received instantaneous Signal-to-Noise Ratio (SNR) is above a threshold value $\gamma_{\text {th }}$.

\section{3-2 Link Models}

In this subsection, we consider the four link models and the outage probability is also derived for each model. First, let us denote $T$ as the set of transmitters and $R$ as the set of receivers.

\section{3-2-1 Point to Point Link}

This case considers only a transmitter $\left(T=\left\{t_{1}\right\}\right)$ sending the packet to a single receiver $\left(R=\left\{r_{1}\right\}\right)$. Using the probability density function (pdf) of the exponential variable $\left|h_{i, j}\right|^{2}$ with parameter $d_{i, j}^{\beta}$, the outage probability of a point-to-point link is given as [9], Eq. [4]

$$
\begin{aligned}
P_{t_{1}-r_{1}}^{\mathrm{P} 2 \mathrm{P}} & =\operatorname{Pr}\left(\gamma\left|h_{t_{1}-r_{1}}\right|^{2}<\gamma_{t h}\right)=\operatorname{Pr}\left(\left|h_{t_{1}-r_{1}}\right|^{2}<\frac{\gamma_{t h}}{\gamma}\right) \\
& =1-\exp \left(-d_{t_{1}-r_{1}}^{\beta} \rho\right)
\end{aligned}
$$

where $\gamma\left|h_{t_{1}-r_{1}}\right|^{2}$ is the received SNR at the receiver with $\gamma=P / N_{0}$ and $\rho=\gamma_{t h} / \gamma=\gamma_{t h} N_{0} / P$.

\section{3-2-2 Point to Multi-Point Link}

In this case, $T=\left\{t_{1}\right\}$ and $R=\left\{r_{1}, r_{2}, \ldots, r_{m}\right\}$ with $m>1$, hence the probability that all links $t_{1}-r_{j}(j=1,2, . ., m)$ are outage is given by

$$
P_{t_{1}-R}^{\mathrm{P} 2 \mathrm{M}}=\prod_{j=1}^{m} \operatorname{Pr}\left(\left|h_{t_{1}, r_{j}}\right|^{2}<\rho\right)=\prod_{j=1}^{m}\left(1-\exp \left(-d_{t_{1}, r_{j}}^{\beta} \rho\right)\right)
$$

Let $D$ denote the set of nodes that successfully decode the packet received from the transmitter $t_{1} . D$ is obviously a subset of $R$ and the size of $D,|D|$, varies from 0 to $m$. If $|D|=0$, no node in $R$ can decode correctly and the probability of this event is calculated according to Eqn. (4). If $|D|>0$, the probability of this event is given as

$$
P_{T-R / D}^{\mathrm{P} 2 \mathrm{M}}=\prod_{r_{j} \in D}\left(\exp \left(-d_{t_{1}, r_{j}}^{\beta} \rho\right)\right) \prod_{r_{j} \notin D}\left(1-\exp \left(-d_{t_{1}, r_{j}}^{\beta} \rho\right)\right)
$$

Note that there are $C_{m}^{n}=\frac{m !}{(m-n) ! n !}$ possibilities for the case of $|D|=n, n \leq m$, and hence there are $2^{m}$ possibilities for $D$. In the case where all members of the set $R$ decode successfully, $(|D|=m)$, the probability that this event occurs, is calculated as

$$
P_{T-R / \mid D}^{\mathrm{P} 2 \mathrm{M}}=\prod_{j=1}^{m} \exp \left(-d_{t_{1}, r_{j}}^{\beta} \rho\right)
$$

\section{3-2-3 Multi-Point to Point Link}


In this case, the transmitters belonging to $T$ ( $T=$ $\left.\left\{t_{1}, t_{2}, \ldots, t_{m}\right\}\right)$ send the same packet to a single receiver $r_{1}$. Then, the receiver $r_{1}$ uses MRC technique to combine all received packets and decodes them. Therefore, the total SNR at the output of the combiner is given as

$$
\gamma_{\text {total }}=\gamma \sum_{j=1}^{m}\left|h_{t_{j}, r_{1}}\right|^{2}
$$

We calculate the outage probability at the receiver $r 1$ using the Moment Generating Function (MGF) to find the CDF of variable $H=\sum_{j=1}^{m}\left|h_{t_{j}, r_{1}}\right|^{2}$. Since each $\left|h_{t_{j}, r_{1}}\right|^{2}$ is exponentially distributed with parameter $d_{t_{j}, r_{1}}^{\beta}$, the MGF of $\left|h_{t_{j}, r_{1}}\right|^{2}$ is $M G F_{\mid h_{t_{j}, r_{1} \mid}{ }^{2}}(s)=\frac{d_{t_{j}, r_{1}}^{\beta}}{d_{t_{i}, r_{1}}^{\beta}+s}$. Due to the independence of $\left|h_{t_{j}, r_{\mathrm{i}}}\right|^{2}$, the MGF of $H$ is given by [10]

$$
M G F_{H}(s)=\prod_{j=1}^{m} \frac{d_{t_{j}, r_{1}}^{\beta}}{d_{t_{j}, r_{\mathrm{i}}}^{\beta}+s}
$$

The parameters $d_{t_{j}, r_{1}}^{\beta}$ are different from each other, so by applying the inverse Laplace Transform to Eqn. (8), the outage probability in this case is calculated by

$$
P_{T-r_{1}}^{\mathrm{M} 2 \mathrm{P}}=\sum_{i=1}^{m} \alpha_{i}\left(1-\exp \left(-d_{t_{i}, r_{1}}^{\beta} \rho\right)\right)
$$

where $\alpha_{i}=\prod_{j=1, j \neq i}^{m} \frac{d_{j, 1}^{\beta}}{d_{j, 1}^{\beta}-d_{i, 1}^{\beta}}$.

\section{3-2-4 Multi-Point to Multi-Point Link}

In this case, $T=\left\{t_{1}, t_{2}, \ldots, t_{m}\right\}, R=\left\{r_{1}, r_{2}, \ldots, r_{n}\right\}$ with $m>1, n>1$ and each node belonging to the set $R$ uses the MRC technique to combine all packets transmitted by members of the set $T$ and decodes them. Let $D$ $(D \subset R)$ denote the set of nodes decoding successfully, the probability for each case of the set $D$ can be given as

$$
P_{T-R / D}^{\mathrm{M} 2 \mathrm{M}}=\prod_{r_{j} \notin D}\left(P_{T-r_{j}}^{\mathrm{M} 2 \mathrm{P}}\right) \prod_{r_{j} \in D}\left(1-P_{T-r_{j}}^{\mathrm{M} 2 \mathrm{P}}\right)
$$

where $P_{T-r_{j}}^{\mathrm{M} 2 \mathrm{P}}$ which is calculated by to Eqn. (9) is the probability that the receiver $r_{j}$ incorrectly decodes the packets.

\section{3-3 End-to-end Outage Probability}

In this subsection, we derive the end-to-end outage probability of the $M$-hop route as shown in Fig. 1. Each hop of the route is assumed to be independent.

\section{3-3-1 The NCRP}

For the NCRP, the end-to-end outage probability can be calculated recursively as follows

$$
P_{N_{1}-N_{M+1}}^{\mathrm{NCRP}}=P_{N_{1}-N_{M+1}}^{\mathrm{P} 2 \mathrm{P}} \times\left(\prod_{t=2}^{M} P_{N_{1}-N_{t}}^{\mathrm{P} \mathrm{P}}+\sum_{D} Q_{D} \times P_{D-N_{M+1}}^{\mathrm{NCRP}}\right)
$$

where $D$ is set of nodes that correctly decode the packet received from the source $N_{1}$ and the size of $D$ varies from 1 to $M$. With $D=\left\{N_{k_{1}}, N_{k_{2}}, \ldots, N_{k_{n}}\right\},(2 \leq$ $\left.k_{1}, k_{2}, \ldots, k_{n} \leq M\right), Q_{D}$ and $P_{D-N_{M+1}}^{\mathrm{NCRP}}$ are given by

$$
\begin{aligned}
& Q_{D}=\prod_{N_{j} \notin D}\left(P_{N_{1}-N_{j}}^{\mathrm{P} \mathrm{P}}\right) \prod_{N_{j} \in D}\left(1-P_{N_{1}-N_{j}}^{\mathrm{P} 2 \mathrm{P}}\right) \\
& P_{D-N_{M+1}}^{\mathrm{NCRP}}= \\
& \begin{cases}P_{N_{k_{1}}-N_{M+1}}^{\mathrm{P} 2 \mathrm{P}}\left(\prod_{j=k_{1}+1}^{M} P_{N_{k_{1}}-N_{j}}^{\mathrm{P} \mathrm{P}}+\sum_{D_{1}} Q_{D_{1}} \times P_{D_{1}-N_{M+1}}^{\mathrm{NCRP}}\right) & \text { if }|D|=1 \\
P_{D-N_{M+1}}^{\mathrm{M} 2 \mathrm{P}}\left(\prod_{j=k_{n}+1}^{M} P_{D-N_{j}}^{\mathrm{M} 2 \mathrm{P}}+\sum_{D_{1}} Q_{D_{1}} \times P_{D_{1}-N_{M+1}}^{\mathrm{NCRP}}\right) & \text { if }|D| \geq 2\end{cases}
\end{aligned}
$$

In Eqn. (13), if the destination cannot decode correctly from the packets received from the nodes of the set $D$, it requests a retransmission from the relays $N_{h}$ $\left(k_{n}+1 \leq h \leq M\right)$ that have decoded successfully. Let us denote $D_{1}$ as the set of these relays. Depending on the size of $D$ and $D_{1}$, the decoding probability set $Q_{D_{1}}$ can be calculated according to one of four link models presented above. For example, if $|D|>1$ and $\left|D_{1}\right|>1, Q_{D_{1}}$ is calculated similarly to Eqn. (10). In addition, $P_{D_{1}-N_{M+1}}^{\mathrm{NCRP}}$ is also given similarly to $P_{D-N_{M+1}}^{\mathrm{NCRP}}$ in Eqn. (13).

However, with the NCRP, each node can store many versions of the packet for decoding if the number of successful relays at the previous stage is large. Therefore, we also propose another protocol, namely $k$-NCRP. In this protocol, if the destination incorrectly decodes the packet, $k$ of the successful relays that are nearest to destination will be chosen to retransmit the packet to the destination. The operation of choosing these $k$ relays can be realized in a distributed manner as follows. Similar to the NCRP protocol, whenever the destination cannot decode the signal correctly, it will generate a NACK message to inform. After receiving the NACK message, relay $N_{M}$ will be the first node to transmit the control message to inform the decoding status. If it decodes correctly, it will transmit an ACK message. Otherwise, it will transmit the NACK message. Similarly, the relays 
$N_{M-1}, N_{M-2}, \cdots, N_{2}$ sequentially will transmit a control message until a sufficient number of $k$ relays are available that can decode the signal successfully. We assume that control signals can be received by all the nodes on the route without errors, so this operation ends whenever the destination and the relays hear sufficient $k$ ACK messages transmitted by $k$ successful relays. When $k$ equals 1 , we have the 1- NCRP, in which only relay nearest to the destination is selected for a retransmission.

\section{3-3-1-1 The 1-NCRP Protocol}

With this scheme, the end-to-end outage probability is calculated by a recursive expression as

$$
P_{N_{1}-N_{M+1}}^{1-\mathrm{NCRP}}=P_{N_{1}-N_{M+1}}^{\mathrm{P} 2 \mathrm{P}} \times\left(\prod_{t=2}^{M} P_{N_{1}-N_{t}}^{\mathrm{P} 2 \mathrm{P}}+\sum_{t=2}^{M} Q_{t} \times P_{N_{t}-N_{M+1}}^{\mathrm{I} \mathrm{NCRP}}\right)
$$

where $Q_{t}=\left\{\begin{array}{l}\left(1-P_{N_{1}-N_{t}}^{\mathrm{P} 2 \mathrm{P}}\right) \prod_{k=t+1}^{M} P_{N_{1}-N_{k}}^{\mathrm{P} 2 \mathrm{P}} \quad \text { if } M \geq t+1 \\ \left(1-P_{N_{1}-N_{M}}^{\mathrm{P} 2 \mathrm{P}} \text { if } M=t\right.\end{array}\right.$.

\section{3-3-1-2 The k-NCRP Protocol}

In this case, the end-to-end outage probability of an $M$-hop route is also expressed recursively as

$$
P_{N_{1}-N_{M+1}}^{k \text {-NCRP }}=P_{N_{1}-N_{M+1}}^{\mathrm{P} 2 \mathrm{P}} \times\left(\prod_{t=2}^{M} P_{N_{1}-N_{t}}^{\mathrm{P} 2 \mathrm{P}}+\sum_{D} Q_{D} \times P_{D-N_{M+1}}^{k-\mathrm{NCRP}}\right)
$$

Assume that $I$ is set of nodes that decode correctly the packet and $D$ is set of selected relays $(D \subset I)$. As mentioned above, if $I=\left\{N_{t_{1}}, N_{t_{2}}, \ldots, N_{t_{n}}\right\}$, then $k$ chosen relays are determined as

$$
D= \begin{cases}I ; & \text { if }|I|=n \leq k \\ \left\{N_{t_{n+1-k}}, N_{t_{n+2-k}} \ldots, N_{t_{n}}\right\} ; & \text { if }|I|=n>k\end{cases}
$$

Therefore, $Q_{D}$ is given by

$$
Q_{D}= \begin{cases}\prod_{N_{j} \notin I}\left(P_{N_{1}-N_{j}}^{\mathrm{P} 2 \mathrm{P}}\right) \prod_{N_{j} \in I}\left(1-P_{N_{1}-N_{j}}^{\mathrm{P} 2 \mathrm{P}}\right) & \text { if }|I|=n \leq k \\ \prod_{\substack{N_{j} \notin I \\ N_{j} \geq N_{k_{1}}}}\left(P_{N_{1}-N_{j}}^{\mathrm{P} 2 \mathrm{P}}\right) \prod_{\substack{N_{j} \in I \\ N_{j}=N_{k_{1}}}}\left(1-P_{N_{1}-N_{j}}^{\mathrm{P} 2 \mathrm{P}}\right) & \text { if }|I|=n>k\end{cases}
$$

Next, the outage probability $P_{D-N_{M+1}}^{k \text {-NCRP }}$ is calculated similarly to $P_{D}^{k \text {-NCRP }}$ in Eqn. (13).

\section{3-3-2 Conventional Multi-hop Transmission Protocols}

\section{3-3-2-1 The Direct Transmission Protocol (DT)}

In this protocol, the packet is relayed hop-by-hop and the receiving node only decodes the packet received from its previous transmitting node. Due to the independence of each hop, the end-to-end outage probability of an $M$-hop route is calculated as

$$
P_{N_{1}-N_{M+1}}^{D T}=1-\prod_{j=1}^{M}\left(1-P_{N_{j}-N_{j+1}}^{\mathrm{P} P}\right)=1-\prod_{j=1}^{M}\left(1-\exp \left(-d_{N_{j}, N_{j+1}}^{\beta} \rho\right)\right)
$$

\section{3-3-2-2 The Novel Direct Transmission Protocol (No- DT)}

For a fair comparison between the proposed protocols and the direct transmission protocols, we consider the novel direct transmission protocol (NoDT). In this protocol, if the destination cannot successfully decode the packet received from the node $N_{j}$, the node $N_{j+1}$ will retransmit it to the destination if it decodes correctly. If node $N_{j+1}$ fails to decode, the signal will be dropped. In this protocol, the end-to-end outage probability can be calculated recursively as follows:

$$
P_{N_{i}-N_{j}}^{\mathrm{NoDT}}=P_{N_{i}-N_{j}}^{\mathrm{P} 2 \mathrm{P}} \times\left(P_{N_{i}-N_{i+1}}^{\mathrm{P} 2 \mathrm{P}}+\left(1-P_{N_{i}-N_{i+1}}^{\mathrm{P} 2 \mathrm{P}}\right) \times P_{N_{i+1}-N_{j}}^{\mathrm{NoDT}}\right)
$$

In Eqn. (19), if $i=1$ and $j=M+1$, we obtain the end-to-end outage probability for an $M$-hop route using the NoDT protocol.

\section{Simulation Results}

In this section, we present Monte Carlo simulation results to verify the theoretical results and to compare the performances of the protocols discussed in the previous sections. In all of the simulations, we assume that the distance between two adjacent nodes on the established route is equal to 1 and the path loss exponent equals to 3.

Figs. 5, 6 present the end-to-end outage probability for the NCRP, 2-NCRP, 1-NCRP, NoDT, and DT protocols as a function of SNR in $\mathrm{dB}$. In these figures, the direction of the arrows indicates the decreasing of the threshold value $\gamma_{t h}$ and the number of hops $M$, respectively. In Fig. 5, we consider the outage performance of the protocols in the 8-hop networks with the different threshold values of $\gamma_{t h}$, i.e., 2.5 and 3. This figure shows that the NCRP protocol obtains the best performance at high SNR, while the DT protocol gets the worst performance. In addition, the performance of all the protocols is better if the threshold value $\gamma_{t h}$ decreases. In Fig. 6, we investigate the effect of the number of hops on the outage performance. In this figure, the threshold 


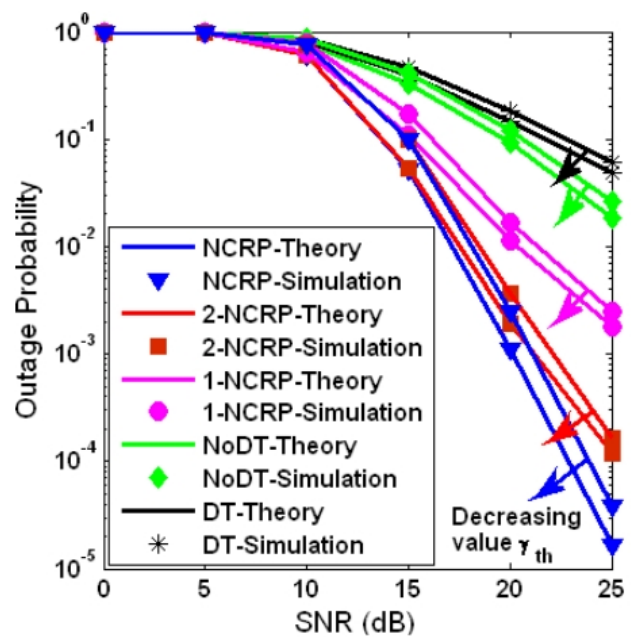

Fig. 5. The end-to-end outage probability as a function of the SNR with different threshold $\gamma_{t h}$.

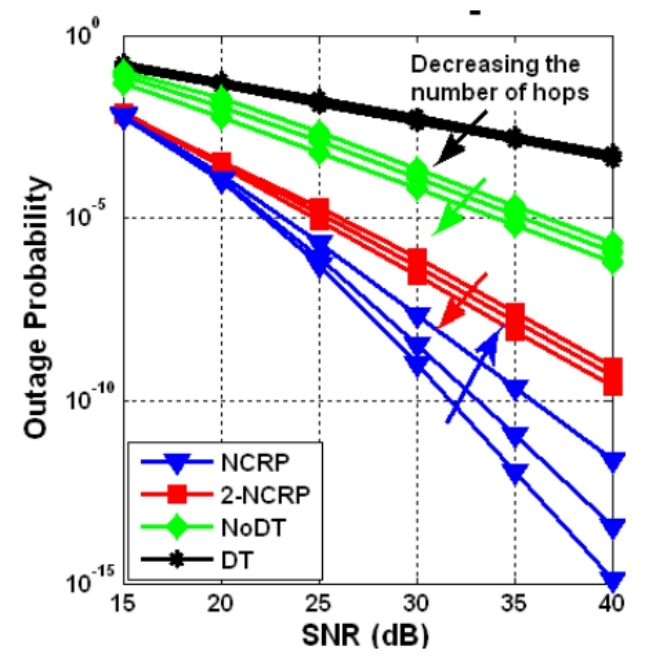

Fig. 6. The end-to-end outage probability as a function of SNR with the different number of hops.

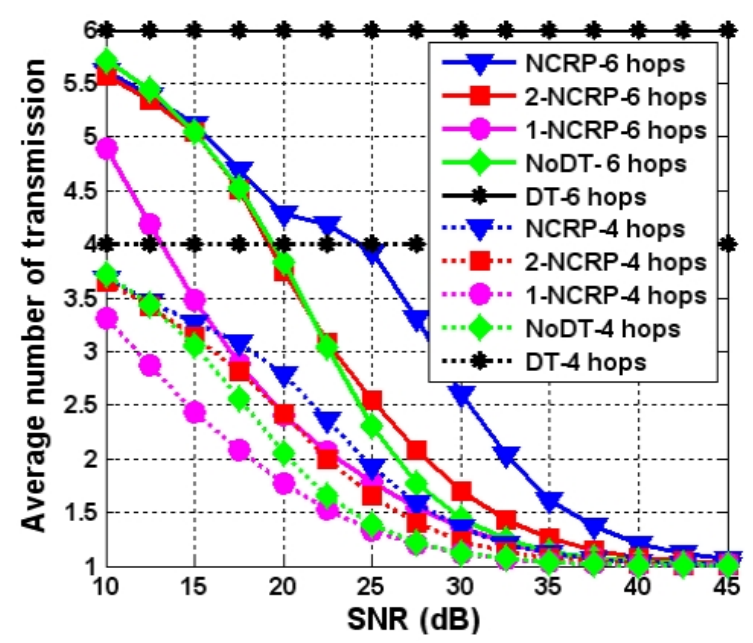

Fig. 7. The average number of transmissions as a function of SNR with different number of hops. value is fixed at 1 , the number of hops varies from 4 to 6 , and the end-to-end outage probability is presented by the theoretical results. As we can see, the performance of the 2-NCRP protocol is better than that of the NoDT protocol but worse than that of the NCRP protocol. In addition, unlike the other protocols, the NCRP protocol achieves better performance when the number of hops increases.

In Fig. 7, we present the average number of transmission as a function of the SNR when the threshold value is fixed at 2 . The number of transmissions of the DT equals the number of hops, while the number of transmissions for the remaining protocols is smaller due to the skipped transmissions at the intermediate relays. Among the presented protocols, the 1-NCRP protocol uses least number of transmissions, while the NCRP protocol uses more transmissions compared to the 2NCRP and NoDT protocols. Furthermore, because the data transmission is realized by TDMA technique, reducing the number of transmission also means reducing the delay time and power consumption. Thus, the proposed protocol can save power, compared to the DT protocol.

\section{Conclusion}

In this paper, we proposed a novel cooperative routing protocol (NCRP) for wireless networks. We also derived the exact expressions of the end-to-end outage probability for the NCRP protocol and verified this by simulations. The simulation results showed that the NCRP protocol improves the performance at the high SNR region when compared with the NoDT and DT protocols. The proposed protocol can also reduce power consumption due to the skipped transmissions at the intermediate relays. For future work, as presented in [11], we expect to realize the proposed protocol using wireless receiving devices using $\mathrm{A} / \mathrm{D}$ converters and FIR filters and etc.

This work was supported by the 2012 Research Fund of University of Ulsan.

\section{References}

[1] J. N. Laneman, D. N. C. Tse, and G. W. Wornell, "Cooperative diversity in wireless networks: efficient protocols and outage behavior," IEEE Trans. Inform. Theory, vol. 50, no. 12, pp. 3062-3080, 2004.

[2] T. E. Hunter, A. Nosratinia, "Diversity through coded cooperation," IEEE Trans. on Wireless Commu., vol. 
5, no. 2, pp. 283-289, 2006.

[3] A. Bletsas, A. Khisti, D. P. Reed, and A. Lippman, "A simple cooperative diversity method based on network path selection," IEEE Journal on Selected Areas in Commun., vol. 24, no. 3, pp. 659-672, 2006.

[4] G. K. Karagiannidis, "Performance bounds of multihop wireless communications with blind relays over generalized fading channels," IEEE Trans. Wire. Coтти., vol. 5, no. 3, pp. 498-503, 2006.

[5] J. Boyer, David D. Falconer, and H. Yanikomeroglu, "Multihop diversity in wireless relaying channels," IEEE Trans. on Commun., vol. 52, no. 10, pp. 18201830, 2004.

[6] Z. Yi, Il-Min Kim, "Relay ordering in a multi-hop cooperative diversity network," IEEE Trans. on Coтmи., vol. 57, no. 9, pp. 2590-2596, 2009.

[7] G. Farhadi, N. Beaulieu, "Fixed relaying versus se-

\section{Tran Trung Duy}

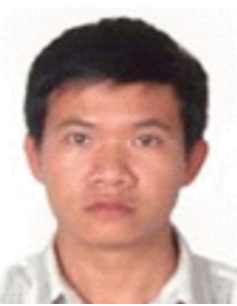

received Telecommunications Engineering from Ho Chi Minh City University of $\mathrm{Te}$ the B.E. degree in Electronics and technology, Vietnam, in 2007. He is currently working toward the Master degree in the Department of Electrical Engineering, University of Ulsan, Korea. His major research interests are mobile ad-hoc networks, wireless sensor networks, cooperative communications, cooperative routing, cognitive radio, combining techniques. lective relaying in multi-hop diversity transmission systems," IEEE Trans. on Wire. Commun., vol. 58, no. 3, pp. 956-965, 2010.

[8] C. Conne, J. MinChul, Z. Yi, H. Song, and Il-Min Kim, "SER analysis and PDF derivation for multihop amplify-and-forward relay systems," IEEE Trans. on Wire. Commun., vol. 58, no. 8, pp. 24132424, 2010.

[9] T. T. Duy, B. An, and H. -Y. Kong, "A novel cooperative-aided transmission in multi-hop wireless networks," IEICE Trans. on Commun., vol. E93.B, no. 3, pp. 716-720, Mar. 2010.

[10] K. Knight, Mathematical Statistics, Chapman and Hall, 1999.

[11] T. Mimura, "Packet transmission experiments of STBC-based multi-hop cooperative relaying," Соттиnications (ICC), 2011 IEEE International Conference on, pp. 1-5, Jun. 2011.

Jong-Soo Kim

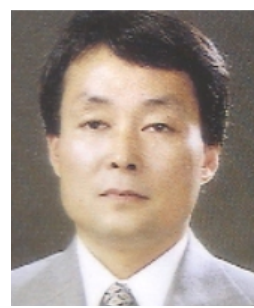

received his Ph.D. degree from the University of Alabama in Hunstville in 1994. Currently, he is a professor in the School of Electrical Engineering, University of Ulsan, Ulsan, Republic of Korea. His current research interests include lowpower circuits, mixed signal circuits, $\mathrm{A} /$ $\mathrm{D}$ converter circuits design, and high-level synthesis. 